This article was retracted on 18 January 2022, due to the authors' offer.

\title{
Long non-coding RNA TUG1 promotes the osteogenic differentiation of bone marrow mesenchymal stem cells by regulating the AMPK/mTOR/autophagy pathway
}

\author{
Ding-gui Lu, Mei-jiao Lu, Shun-han Yao, Jia-jie Lin, Su Luo, Ji-hua WeI, and Yu-jin Tang \\ Department of Orthopedics, Affiliated Hospital of Youjiang Medical University for Nationalities, Baise, 533000, Guangxi, China \\ (Received 24 March 2021; and accepted 27 May 2021)
}

\begin{abstract}
Promoting the differentiation of bone marrow mesenchymal stem cells (BMSCs) into osteoblasts is an effective strategy against osteoporosis. Long non-coding RNAs are closely implicated in BMSC osteogenic differentiation. The present study explored the expression pattern and biological role of taurine upregulated gene 1 (TUG1) in osteogenic differentiation. The expressions of TUG1 and osteogenic markers following the osteogenic induction of BMSCs were detected. The functional relevance of TUG1 was evaluated by performing gain- and loss-of-function tests. Inhibitors of AMP-activated protein kinase (AMPK) autophagy were applied to ascertain the effects of TUG1 on the osteogenic differentiation of BMSCs. TUG1 expression increased during the osteogenic differentiation of BMSCs. The overexpression of TUGl was promoted, whereas the knockdown of TUG1 was suppressed, by BMSC osteogenic differentiation Mechanically, TUG1 promoted the osteogenesis of BMSCs via the AMPK-mammalian target of rapamycin (mTOR)-autophagy signaling pathway. Blocking AMPK and autophagy could abrogate the osteogenic role of TUG1 in BMSCs. These results demonstrated that TUG1 promoted the osteogenic differentiation of BMSCs by regulating the AMPK/mTOR/autophagy axis, suggesting that targeting TUG1 may be a potential therapy for osteoporosis.
\end{abstract}

\section{INTRODUCTION}

Osteoporosis is a severe metabolic bone disorder in elderly people that occurs due to the imbalance between bone formation and resorption. A family history of fractures, aging, and smoking are considered to be risk factors for osteoporosis. The key to the prevention of osteoporosis is to restore the normal bone content. Patients with osteoporosis are usually

Address correspondence to: Ji-hua Wei, Department of Orthopedics, Affiliated Hospital of Youjiang Medical University for Nationalities, Baise, 533000, Guangxi, China

E-mail: 1261290953@qq.com

Yu-jin Tang, Department of Orthopedics, Affiliated Hospital of Youjiang Medical University for Nationalities, Baise, 533000, Guangxi, China

E-mail: tyj196609@163.com treated with calcium supplements and hormone replacement therapy (Armas and Recker, 2012; Coughlan and Dockery, 2014; Compston et al., 2019). Bone marrow mesenchymal stem cells (BMSCs) are multipotent stromal cells that can differentiate into osteoblasts, adipocytes, chondrocytes, etc. The osteogenic differentiation of BMSCs is critical in bone remodeling and the decreased capability of differentiation may be the pathophysiological basis of osteoporosis (Jin et al., 2019; Pierce et al., 2019). Therefore, it is important to elucidate the molecular mechanism of BMSC osteogenic differentiation.

Long non-coding RNAs (lncRNAs) are noncoding transcripts that are longer than 200 nucleotides (Quinn and Chang, 2016). IncRNAs play diverse roles in physiological and pathological processes, such as proliferation, apoptosis, differentiation, migration, and autophagy (Kopp and Mendell, 2018). Deregulated expression of lncRNA has been ob- 
served in various human diseases, such as carcinogenesis, cardiovascular disease, and bone-related disorders (Ørom and Shiekhattar, 2013). Additionally, the functional relevance of lncRNAs in the osteogenic differentiation of BMSCs has been studied in recent years. For instance, GAS5 promotes the osteogenesis of BMSCs by functioning as a competing endogenous RNA for miR-135a-5p (Wang et al., 2019). In addition, the DEP domain containing mammalian target of rapamycin (mTOR) interacting protein accelerates osteogenic differentiation by inhibiting the lncRNA MEG3-mediated activation of bone morphogenetic protein 4 (BMP4) signaling (Chen et al., 2018). Taurine upregulated gene 1 (TUG1), located on chromosome 22q12.2, has been widely studied in human malignancies ( $\mathrm{Li}$ et al., 2016). Knockdown of TUG1 retards osteoblast differentiation in calcific aortic valve disease $(\mathrm{Yu}$ et al., 2018). However, whether TUG1 can promote the osteogenic differentiation of BMSCs is currently unknown.

Autophagy is an intracellular degradation process by which cytoplasmic materials are transported to lysosomes for degradation and the content is recycled (Dikic and Elazar, 2018). Autophagy is important for normal physiological behaviors, and autophagic disorder may lead to various diseases, such as cancer, inflammatory disease, and central nervous system malfunction (Levine and Kroemer, 2008). Additionally, autophagy is involved in osteogenic differentiation and bone formation (Yang et al., 2020). Furthermore, AMP-activated protein kinase (AMPK) and mTOR are crucial for autophagy (Kim and Guan, 2015; Herzig and Shaw, 2018). IncRNAs may activate the AMPK signaling pathway and thus, promote autophagy upon energy deficiency (Li et al., 2018). However, whether IncRNA TUGI can regulate the osteogenic induction of BMSCs yia the AMPK/mTOR/autophagy axis is currently unknown. Using gain- and loss-of-function tests, the present study detected the expression and functional relevance of IncRNA TUG1 during osteogenic induction in BMSCs. Furthermore, we explored whether lncRNA TUG1 regulated osteogenesis via activation of the AMPK/mTOR/autophagy axis.

\section{MATERIALS AND METHODS}

BMSC isolation and culture. BMSCs were isolated from the femora of one-week-old Sprague-Dawley rats. Briefly, collected bone marrow cells were cultured in Dulbecco's modified Eagle's medium (DMEM; Invitrogen, Carlsbad, CA, USA) supple- mented with $10 \%$ fetal bovine serum (Gibco, Grand Island, NY, USA) at $37^{\circ} \mathrm{C}$ with $5 \% \mathrm{CO}_{2}$. The medium was replaced every two days. Cells at passage three were used in this study.

Transfections. Transfections of plasmid DNA or siRNAs were performed using the Lipofectamine 3000 (ThermoFisher Scientific, Inc., Waltham, MA, USA) according to the manufacturer's instructions. BMSCs were seeded onto a 24-well plate and transfected with pcDNA3.1-TUG1, TUG1 siRNA, or their negative controls (GeneChem, Shanghai, China). Then, $48 \mathrm{~h}$ later, cells were harvested for further experiments.

Osteogenic differentiation induction. To induce osteoblastic differentiation, BMSCs at $90 \%$ confluence were cultured in osteogenesis induction medium containing DMEM supplemented with $10 \%$ fetal bovine serum, $100 \mu \mathrm{M}$ L-asoorbic acid, $10 \mathrm{mM}$ of $\beta$ glycerophosphate, and $10 \mathrm{nM}$ of dexamethasone at $37^{\circ} \mathrm{C}$ with $5 \% \mathrm{CO}_{2}$. The medium was changed every two days and BMSCs were induced for 21 days.

Quantitative real time polymerase chain reaction ( $q R T-P C R)$. Total RNAs from BMSCs were isolated using TRIzol reagent (Invitrogen) and reverse transcribed into cDNA using the PrimeScript RT Master Mix (TaKaRa, Dalian, China). PCR reactions were conducted using the SYBR Green PCR master mix (Roche, Basel, Switzerland) to detect the expression of the target genes using the 7900 HT Sequence Detection System (ABI, USA). GAPDH was used as the internal control. The primers used were TUG1: forward 5'-GGCAACAACTCACCCAGCACTG-3', reverse 5'-AGGAGGCACGGGACGTAGTTC-3'; RUNX family transcription factor 2 (RUNX2): forward 5'-CCAAGTGGCCAGTTCAACG-3', reverse 5'-GGGATGAGGAATGCGCCCTA-3'; bone sialoprotein (BSP): forward 5'-CCGGGAGAACAATCC GTGCC-3', reverse 5'-AAAGCACTCGCCATCCCC AA-3'; osteocalcin (OCN): forward 5'-CAGGTGC AAAGCCCAGCGAC-3', reverse 5'-TGGGGCTCC AAGTCCATTGTT-3'; and, collagen type I, alpha 1 (COL1A1): forward 5'-CATGAGCCGAAGCTAAC CC-3', reverse 5'-CTCCTATGACTTCTGCGTCT GG-3'.

Immunofluorescence staining. For immunofluorescence staining, BMSCs were infected with red fluorescent protein (RFP)-LC3-labeled lentivirus, an autophagic flux reporter comprised of LC3 protein fused with RFP. Red fluorescent signals in the cyto- 
plasm indicated autophagosomes. Nuclei were stained with 4',6-diamidino-2-phenylindole in the dark for 10 min. The LC3+ puncta numbers were examined using a fluorescence microscope (Leica, Germany).

Western blot. Proteins from BMSCs were extracted using RIPA Lysis Buffer containing protease inhibitors (Sigma-Aldrich, St. Louis, MO, USA). Protein concentrations were analyzed using the Bradford colorimetric assay. Then, $20 \mu \mathrm{g}$ of protein per lane was separated using sodium dodecyl sulfate polyacrylamide gel electrophoresis (SDS-PAGE), transferred to a polyvinylidene fluoride (PVDF) membrane, and blocked in skim milk at room temperature for $1 \mathrm{~h}$. The membranes were incubated with the primary antibodies against LC3A (1 : 1000; Abcam, Cambridge, UK), LC3B (1 : 1000; Abcam), RUNX2 (1: 1000; Abcam), BSP (1:1000; Abcam), OCN (1: 1000; Abcam), and COL1A1 (1: 1000; Abcam). After horseradish peroxidase-labeled secondary antibodies were added at $37^{\circ} \mathrm{C}$ for $1 \mathrm{~h}$, the blots were visualized with the ECL detection system. Protein bands were quantified with Quantity One software (Bio-Rad Laboratories, Hercules, USA) for determination of the $\mathrm{LC} 3 \mathrm{~B} / \mathrm{A}$ ratio.

Alizarin Red staining. Alizarin Red staining was used to detect the osteoblast mineralization 14 days after osteogenic induction. Cells were washed with PBS, fixed with ethanol for $30 \mathrm{~min}$ and stained with Alizarin Red S (Sigma-Aldrich) for $15 \mathrm{~min}$ at room temperature. Cells were washed three times with distilled water and then observed under a light microscope.

Alkaline phosphatase (ALP) staining. Then, 14 days after osteogenic induction, cells were washed with PBS, fixed with ethanol for $30 \mathrm{~min}$ and stained with ALP staining solution (Sigma-Aldrich) in the dark for $15 \mathrm{~min}$ at room temperature. Cells were washed three times with distilled water and then observed under a light microscope (Olympus, Japan).

Enzyme-linked immunosorbent assay. Then, 14 days after osteogenic induction, cells were washed with PBS and the ALP activity was detected using the ALP assay kit (Sigma-Aldrich) according to the manufacturer's protocol.

Statistical analysis. SPSS 20.0 software (SPSS Inc., Chicago, IL, USA) was used for statistical analysis. Experimental data are expressed as mean \pm standard deviation. Comparisons between multiple groups

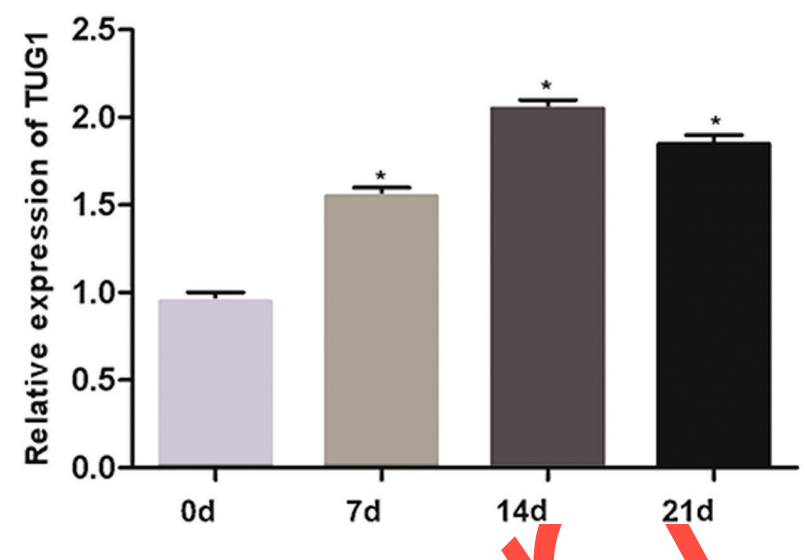

Fig. 1 TUG1 expression is elevated during the osteogenic differentiation of bone marrow mesenchymal stem cells (BMSCs). BMSCs were induced to osteoblast differentiation for 7,14 , and 21 days. GRT-PCR was used to detect the expression of TUG1 during the osteogenic differentiation of BMSCs. ${ }^{*} P<0.05$.

were made using ANOVA. A p-value $<0.05$ was regarded as statistically significant.

\section{RESULTS}

TUG1 expression is elevated during the osteogenic differentiation of $B M S C S$

To establish the functional relevance of TUG1 in BMSCs, we first evaluated its expression pattern during the induction of the osteogenic differentiation of BMSCs. TUG1 expression showed a significant increase at 7, 14, and 21 days following the induction of osteogenic differentiation (Fig. 1), suggesting that TUG1 may play a regulatory role during this process.

TUG1 overexpression promotes the osteogenic differentiation of BMSCs

Next, we applied gain- and loss-of-function tests to explore the biological effects of the lncRNA of TUG1 during the osteogenic induction of BMSCs. First, 14 days after osteogenic induction, ALP staining and activity were found to be suppressed in TUG1-silenced BMSCs (Fig. 2A, B). Conversely, the overexpression of TUG1 significantly enhanced ALP staining and activity in BMSCs (Fig. 2A, B). Moreover, we explored the effect of TUG1 on matrix mineralization by performing Alizarin Red staining and studied the expression of the key osteogenic markers by performing qRT-PCR. Alizarin Red staining showed that the depletion of TUG1 attenuated the intensity of Alizarin Red staining, and its overexpression led to aggravated matrix mineraliza- 

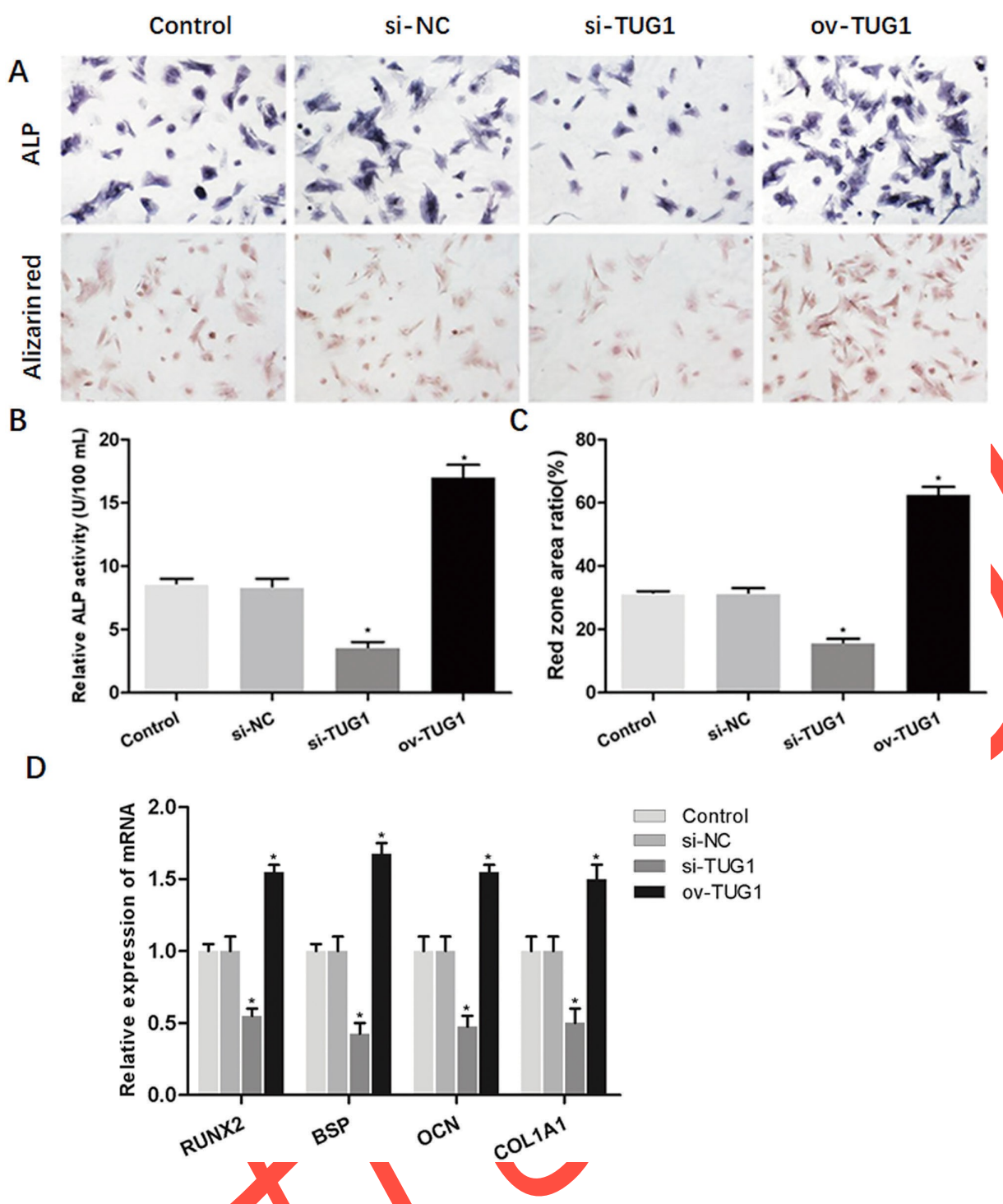

Fig. 2 TUG1 overexpression promotes the osteogenic differentiation BMSCs. BMSCs were transfected with pcDNA3.1-TUG1, TUG1 siRNA, or their negative controls, then induced to osteoblast differentiation for 14 days. (A) ALP activity staining and Alizarin Red staining were performed on day 14 after induction. (B) ALP activity was detected. (C) Quantification of the red zone ratio of Alizarin Red staining. (D) qRT-PCR was used to determine the mRNA expression of RUNX2, BSP, $\mathrm{OCN}$ and $\mathrm{COL} 1 \mathrm{~A} 1 .{ }^{*} P<0.05$.

tion in BMSCs (Fig. 2A, C). Additionally, the mRNA expressions of the osteogenic-related markers (RUNX2, BSP, OCN, and COL1A1) were decreased in TUG1-silenced BMSCs (Fig. 2D). However, the TUG1-overexpressed BMSCs showed higher levels of osteogenic-related markers (Fig. 2D). Taken together, these results showed that TUG1 may promote the osteogenic differentiation of BMSCs.

TUG1 promotes the osteogenic differentiation of BMSCs via autophagy

Furthermore, we measured the expression of autoph- agy markers, i.e., the $\mathrm{LC} 3 \mathrm{~B} / \mathrm{A}$ ratio, in TUG1-overexpressed or silenced BMSCs. At 14 days after osteogenic induction, western blot analysis showed that the depletion of TUG1 decreased the LC3B/A ratio, while the overexpression of TUG1 increased the $\mathrm{LC} 3 \mathrm{~B} / \mathrm{A}$ ratio in BMSCs (Fig. 3A). Additionally, immunofluorescence staining revealed that the number of autophagosomes decreased in TUG1-silenced BMSCs (Fig. 3B). However, the TUG1-overexpressed BMSCs exhibited an increased number of autophagosomes 14 days after the osteogenic induction of BMSCs (Fig. 3B). To ascertain the role of 
A
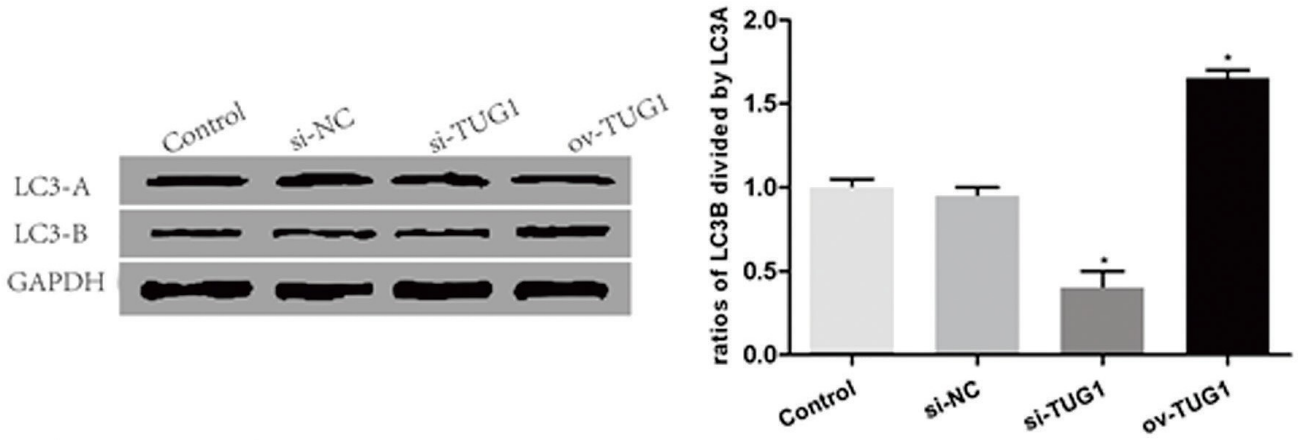

B
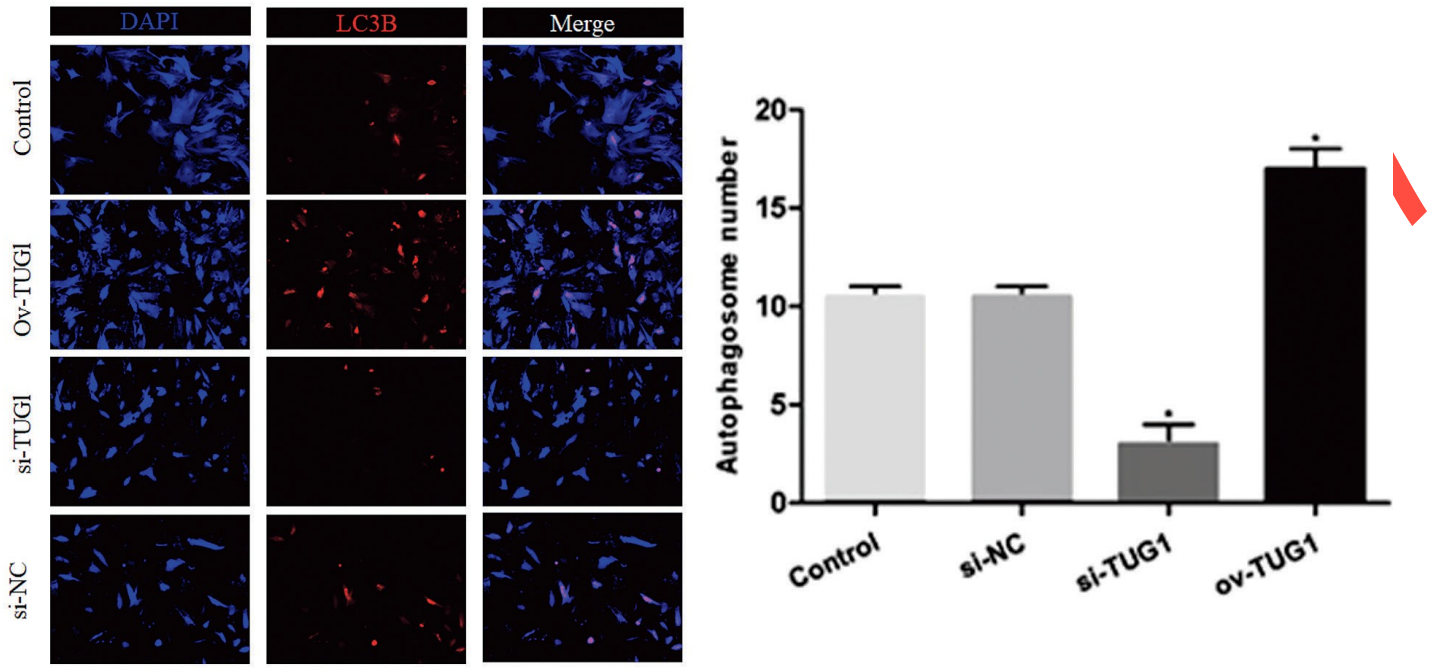

C
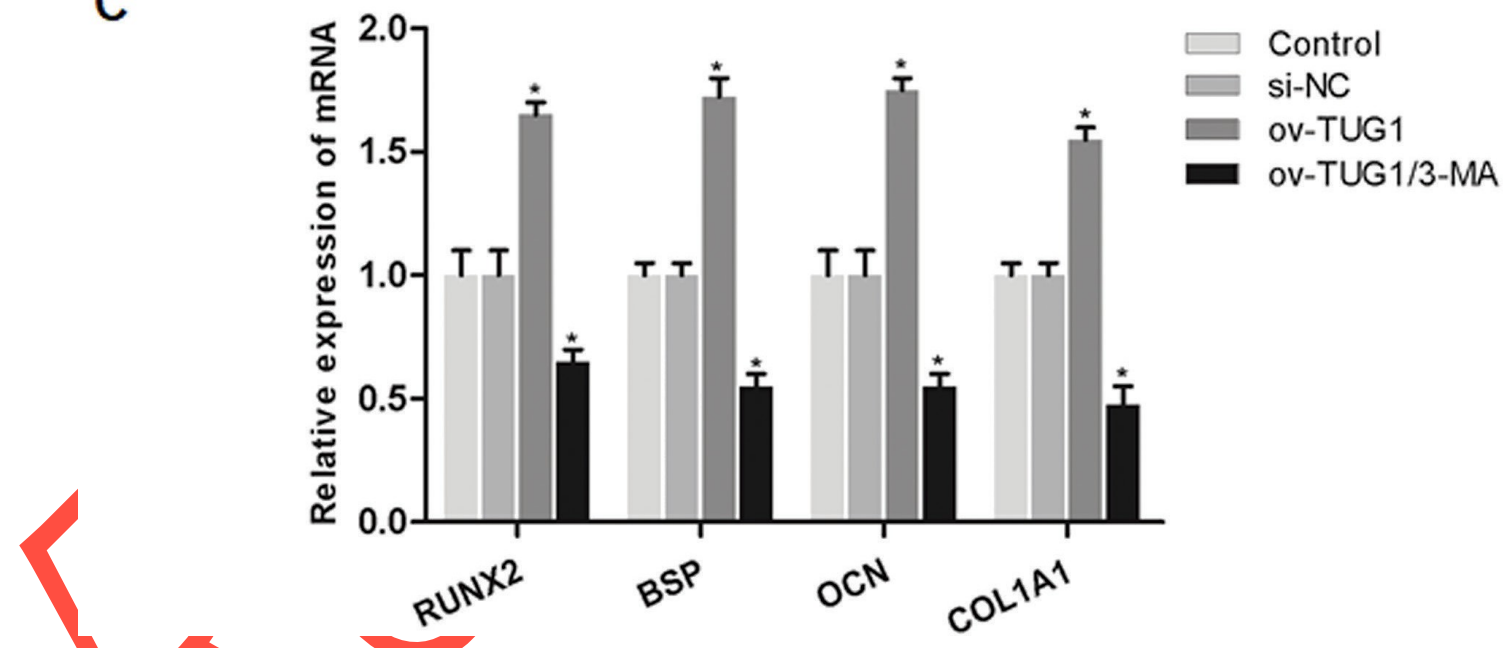

Fig. 3 TUG1 promotes the osteogenic differentiation of BMSCs via autophagy. BMSCs were transfected with pcDNA3.1-TUG1, TUG1 SiRNA, or their negative controls, then induced to osteoblast differentiation for 14 days. (A) Western blot was performed to determine the expression of LC3-A and LC3-B. The ratio of LC3B/A was then calculated. (B) Autophagosomes were detected using immunofluorescence staining, then the number of autophagosomes was calculated. (C) The autophagy inhibitor 3-methyladenine was added to TUG1-overexpressed BMSCs, then cultured for 14 days after osteogenic induction. qRT-PCR was used to determine the mRNA expression of RUNX2, BSP, OCN and COL1A1. ${ }^{*} P<0.05$.

autophagy in TUG1-mediated osteogenic induction, the autophagy inhibitor 3-methyladenine (3-MA) was added to TUG1-overexpressed BMSCs. Conse- quently, the overexpression of TUG1 promoted the expression of osteogenic-related markers, including RUNX2, BSP, OCN, and COL1A1 (Fig. 3C). How- 

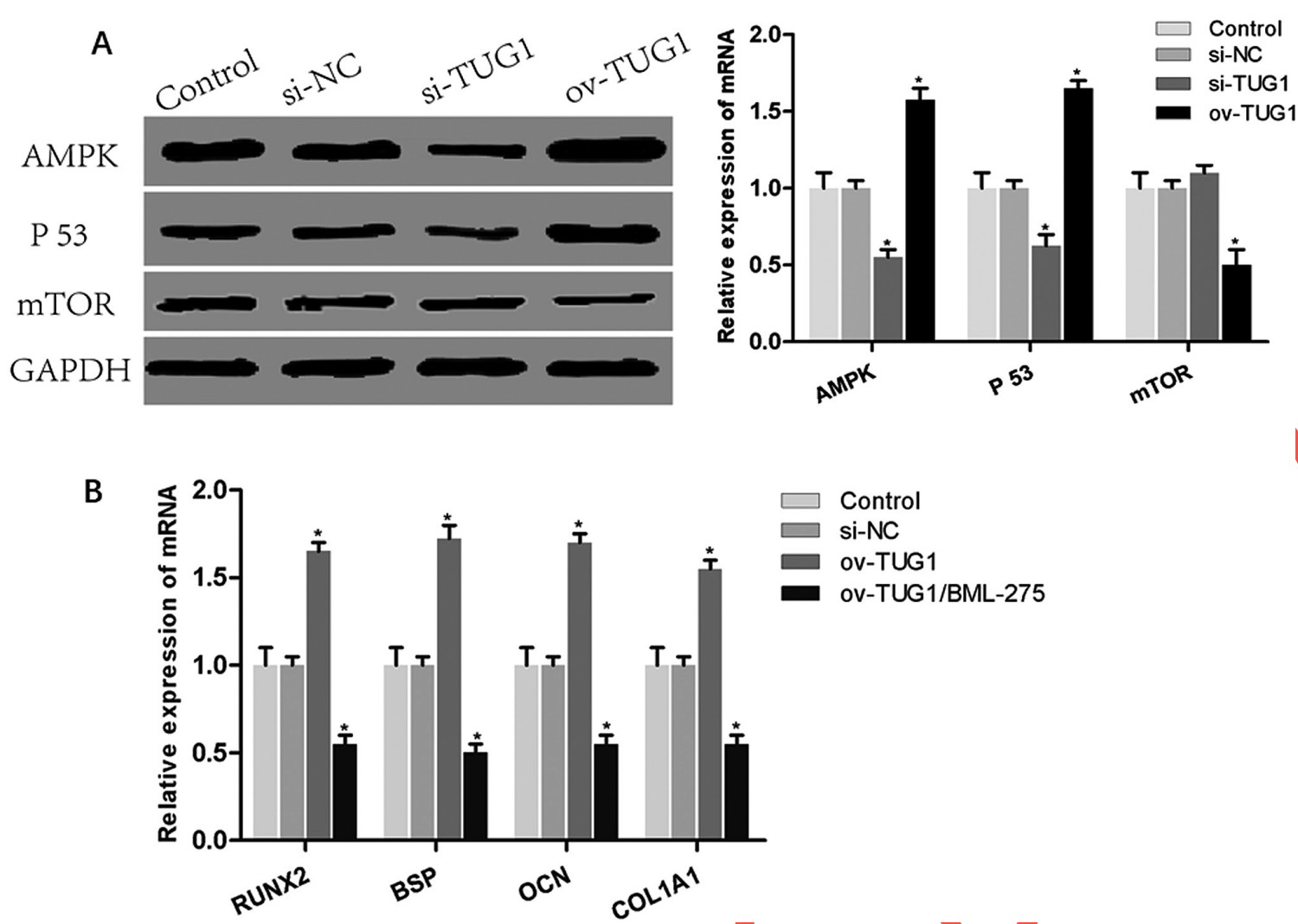

Fig. 4 TUG1 promotes the osteogenesis of BMSCs via AMPK/mTOR/autophagy signaling pathway. BMSCs were transfected with pcDNA3.1-TUG1, TUG1 siRNA, or their negative controls, then induced to osteoblast differentiation for 14 days. (A) Western blot was performed to determine the expression of AMPK, P53, and mTOR. (B) The AMPK inhibitor BML-275 was added to the TUG1-overexpressed BMSCs, then cultured for 14 days after osteogenic induction. qRT-PCR was used to determine the mRNA expression of RUNX2, BSP, OCN and COL1A1. ${ }^{*} P<0.05$.

ever, co-administration with 3-MA abolished the osteogenic role of TUG1 in BMSCs, 14 days after osteogenic induction (Fig. 3C). These results demonstrated that TUG1 promotes the osteogenic differentiation of BMSCs via the regulation of autophagy.

\section{TUG1 promotes the osteogenesis of BMSCS yia the} AMPK/nTOR/autophagy signaling pathway

We then explored the role of the AMPK/mTOR pathway and autophagy in TUG1-induced osteogenesis in BMSCs. At 14 days after osteogenic induction, western blot showed that TUG1-silenced BMSCs exhibited decreased AMPK and P53 expression, whereas the overexpression of TUG1 significantly increased the expressions of AMPK and P53, and decreased mTOR expression in BMSCs (Fig. 4A). Additionally, the AMPK inhibitor BML-275 was added to TUG1-overexpressed BMSCs and then cultured for 14 days. Consequently, the overexpression of TUG1 increased the expression of osteogenic markers (RUNX2, BSP, OCN, and COL1A1)
(Fig. 4B). Nevertheless, treatment with AMPK inhibitor abrogated the osteogenic effects of TUG1 in BMSCs, 14 days after osteogenic induction (Fig. 4B). Collectively, these results indicated that TUG1 promotes the osteogenic differentiation of BMSCs via the AMPK/mTOR/autophagy axis.

\section{DISCUSSION}

In the present study, we found that TUG1 expression increased during the osteogenic differentiation of BMSCs. Functional assays revealed that TUG1 could promote BMSC osteogenic differentiation via the AMPK/mTOR/autophagy axis.

TUG1, located on chromosome 22q12.2 with a length of $7.1 \mathrm{~kb}$ nucleotides, has been extensively studied in various diseases, including neurodegeneration, carcinogenesis, and osteogenic differentiation. TUG1 promotes osteogenic differentiation of periodontal ligament stem cells (He et al., 2018). In the present study, TUG1 showed a significant increase 
in expression during the osteogenic differentiation of BMSCs. To unravel the functional role of TUG1 in osteogenic induction, gain- and loss-of-function tests were performed in BMSCs. Results showed that the overexpression of TUG1 facilitated osteogenic differentiation, while TUG1 knockdown delayed the osteogenic induction of BMSCs, suggesting a positive regulator of TUG1 during BMSC osteogenic differentiation.

TUG1 exerts its function via different mechanisms. For instance, Zhang et al. (2014) found that TUG1 is associated with a poor prognosis in lung cancer; moreover, the overexpression of TUG1 promotes tumorigenesis and development via the epigenetic regulation of HOXB7. TUG1 contributes to the carcinogenesis of human osteosarcoma by serving as a competing endogenous RNA for miR-9-5p (Xie et al., 2016). Additionally, TUG1 promotes osteoblast differentiation through the miR-204-5p/ Runx2 axis during aortic valve calcification ( $\mathrm{Yu}$ et al., 2018). In the present study, results showed that the overexpression of TUG1 led to an increase in the $\mathrm{LC} 3 \mathrm{~B} / \mathrm{A}$ ratio and the number of autophagosomes. Moreover, TUG1-overexpressed cells exhibited increased levels of AMPK and P53, and decreased levels of mTOR. These results implied that TUG1 may promote the osteogenic differentiation of BMSCs via the regulation of autophagy and AMPK. mTOR pathway.

Autophagy is a conserved self-degradation system that can clear damaged organelles, metabolites, and pathogens to maintain cellular homeostasis during stress conditions. Dysregulated autophagy is closely associated with human health and disease (Levine and Kroemer, 2008; Dikic and Elazar, 2018). The mineralization process of osteoblasts is an important step in bone development and metabolism. Abnormal levels of autophagy have critical implications in bone metabolism, mineralization, bone homeostasis, and osteoporosis (Nollet et al., 2014; Vrahnas et al., 2019). AMPK is a type of serine/threonine protein kinase that is implicated in energy metabolism, cell proliferation, differentiation, and apoptosis (Herzig and Shaw, 2018). Activation of the AMPK pathway promotes osteoblast differentiation and mineralization via the regulation of endothelial nitric oxide synthase and BMP (Zhong et al., 2011). To verify whether TUG1 regulates the osteogenic differentiation of BMSCs via autophagy and the AMPK/ mTOR signaling pathway, the autophagy and AMPK inhibitors were applied to TUG1 overexpressing BMSCs. Results showed that blocking autophagy and the AMPK pathway abrogated the positive role of TUG1 on BMSC osteogenic differentiation.

Recently, Li et al. (2018) found that the activation of AMPK stimulates osteoblast differentiation and mineralization through autophagy induction, as evidenced by the upregulation of LC3 and p-AMPK and increased autophagosome density. Furthermore, mTOR is an atypical serine/threonine protein kinase and a sensor for amino acids and ATP, which plays a gating role in the process of autophagy (Saxton and Sabatini, 2017). The suppression of mTOR could promote autophagy, which, in turn, regulates autophagy-dependent mTOR activity (Yu et al., 2010). Upon the activation of AMPK, mTORC1 dissociates from the unc-51-like autophagy activating kinase 1 complex and promotes the formation of the autophagic vesicle membrane (Yu et al., 2018; Lahivi et al., 2019; Abdrakhmanov et al., 2020). To verify whether the AMPK/mTOR/autophagy axis is involved in TUG1-mediated osteogenic differentiation, the AMPK inhibitor was applied to TUG1-overexpressed BMSCs. Results showed that blocking the AMPK pathway inhibited the positive role of TUG1 on the osteogenic differentiation as well as autophagy level of BMSCs.

In conclusion, the present study demonstrated that the expression of TUG1 was upregulated during the osteogenic differentiation of BMSCs. Mechanically, TUG1 promoted the osteogenic potential of BMSCs by regulating the $\mathrm{AMPK} / \mathrm{mTOR} /$ autophagy axis. These results suggest that TUG1 may be a new therapeutic target for osteoporosis.

\section{cknowledgments}

This study is supported by the Basic Ability Improvement Project of Young and Middle-aged Teachers in Guangxi Universities (2018KY0449) to Dinggui Lu; Self-funded Scientific Research Projects of Health and the Family Planning Commission of the Guangxi Zhuang Autonomous Region (Z20170243) to Ding-gui Lu; Baise Scientific Research and Technology Development Project (20170512) to Dinggui Lu; and Key R\&D Projects in Guangxi (GK A18050008) to Yu-jin Tang.

\section{CONFLICTS OF INTEREST}

We declare that we do not have any commercial or associative interest that represents a conflict of interest in connection with the work submitted. 


\section{REFERENCES}

Abdrakhmanov A, Gogvadze V and Zhivotovsky B (2020) To eat or to die: Deciphering selective forms of autophagy. Trends Biochem Sci 45, 347-364.

Armas LA and Recker RR (2012) Pathophysiology of osteoporosis: new mechanistic insights. Endocrinol Metab Clin North Am 41, 475-486.

Chen S, Jia L, Zhang S, Zheng Y and Zhou Y (2018) DEPTOR regulates osteogenic differentiation via inhibiting MEG3-mediated activation of BMP4 signaling and is involved in osteoporosis. Stem Cell Res Ther 9, 185.

Compston JE, McClung MR and Leslie WD (2019) Osteoporosis. Lancet 393, 364-376.

Coughlan T and Dockery F (2014) Osteoporosis and fracture risk in older people. Clin Med (Lond) 14, 187-191.

Dikic I and Elazar Z (2018) Mechanism and medical implications of mammalian autophagy. Nat Rev Mol Cell Biol 19, 349364.

He Q, Yang S, Gu X, Li M, Wang C, et al. (2018) Long noncoding RNA TUG1 facilitates osteogenic differentiation of periodontal ligament stem cells via interacting with Lin28A. Cell Death Dis 9, 455.

Herzig S and Shaw RJ (2018) AMPK: guardian of metabolism and mitochondrial homeostasis. Nat Rev Mol Cell Biol 19, $121-135$

Jin Z, Chen J, Shu B, Xiao Y and Tang D (2019) Bone mesenchymal stem cell therapy for ovariectomized osteoporotic rats: a systematic review and meta-analysis. BMC Musculoskelet Disord 20, 556.

Kim YC and Guan KL (2015) mTOR: a pharmacologic target for autophagy regulation. $J$ Clin Invest 125, 25-32.

Kopp F and Mendell JT (2018) Functional classification and experimental dissection of long noncoding RNAs. Cell 172, 393-407.

Lahiri V, Hawkins WD and Klionsky DJ (2019) Watch what You (Self-) eat: Autophagic mechanisms that modulate metabolism. Cell Metab 29, 803-826.

Levine B and Kroemer G (2008) Autophagy in the pathogenesis of disease. Cell 132, 27-42.

Li Y, Su J, Sun W, Cai L and Deng Z (2018) AMP-activated protein kinase stimulates osteoblast differentiation and mineralization through autophagy induction. In J J Mol Med 41, 25352544.

Li Z, Shen J, Chan MT and Wu WK (2016) TUG1: a pivotal oncogenic long non-coding RNA of human cancers. Cell Prolif 49, 471-475.

Nollet M, Santucci-Darmanin S, Breuil V, Al-Sahlanee R, Cros C, et al. (2014) Autophagy in osteoblasts is involved in mineralization and bone homeostasis. Autophagy 10, 1965-1977.
Ørom UA and Shiekhattar R (2013) Long noncoding RNAs usher in a new era in the biology of enhancers. Cell 154, 11901193.

Pierce JL, Begun DL, Westendorf JJ and McGee-Lawrence ME (2019) Defining osteoblast and adipocyte lineages in the bone marrow. Bone 118, 2-7.

Quinn JJ and Chang HY (2016) Unique features of long non-coding RNA biogenesis and function. Nat Rev Genet 17, 47-62.

Saxton RA and Sabatini DM (2017) mTOR signaling in growth, metabolism, and disease. Cell 169, 361-371.

Shackelford DB and Shaw RJ (2009) The LKB1-AMPK pathway: metabolism and growth control in tumour suppression. Nat Rev Cancer 9, 563-575.

Vrahnas C, Blank M, Dite TA, Tatarczuch L, Ansari N, et al. (2019) Increased autophagy in EphrinB2-deficient osteocytes is associated with elevated secondary mineralization and brittle bone. Nat Commun 10, 3436.

Wang X, Zhao D, Zhu Y, Dong Y and Liu Y (2019) Long noncoding RNA GAS5 promotes osteogenic differentiation of bone marrow mesenchymal stem cells by regulating the miR135a-5p/FOXO1 pathway. Mol Cell Endocrinol 496, 110534.

Xie CH, Cao YM, Huang Y, Shi QW, Guo JH, et al. (2016) Long non-coding RNA TUG1 contributes to tumorigenesis of human osteosarcoma by sponging miR-9-5p and regulating POU2F1 expression. Tumour Biol 37, 15031-15041.

Yang Y, Lin Z, Cheng J, Ding S, Mao WW, et al. (2020) The roles of autophagy in osteogenic differentiation in rat ligamentum fibroblasts: Evidence and possible implications. FASEB J34, 8876-8886.

Yu C, Li L, Xie F, Guo S, Liu F, et al. (2018) LncRNA TUG1 sponges miR-204-5p to promote osteoblast differentiation hrough upregulating Runx2 in aortic valve calcification. Cardiovasc Res 114, 168-179.

Yu L, McPhee CK, Zheng L, Mardones GA, Rong Y, et al. (2010) Termination of autophagy and reformation of lysosomes regulated by mTOR. Nature 465, 942-946.

u L, Chen Y and Tooze SA (2018) Autophagy pathway: Celluar and molecular mechanisms. Autophagy 14, 207-215.

Zhang EB, Yin DD, Sun M, Kong R, Liu XH, et al. (2014) P53regulated long non-coding RNA TUG1 affects cell proliferation in human non-small cell lung cancer, partly through epigenetically regulating HOXB7 expression. Cell Death Dis 5, e1243.

Zhong X, Xiu LL, Wei GH, Liu YY, Su L, et al. (2011) Bezafibrate enhances proliferation and differentiation of osteoblastic MC3T3-E1 cells via AMPK and eNOS activation. Acta Pharmacol Sin 32, 591-600. 